
estudios sobre publicaciones periódicas". Anclajes, vol. XXV, n. ${ }^{\circ} 3$, septiembre-diciembre 202I, pp. 27-35.

\title{
ANCLAJES: APORTES A LA AGENDA CRÍTICA DE LOS ESTUDIOS SOBRE PUBLICACIONES PERIÓDICAS
}

\section{Verónica Delgado}

Instituto de Investigaciones en Humanidades y

Ciencias Sociales, IdIHCS (UNLP-CONICET)

Facultad de Humanidades y Ciencias de la Educación

Universidad Nacional de La Plata

Argentina

vedelgado@yahoo.com

ORCID: 0003-0659-025I

\section{Geraldine Rogers}

Instituto de Investigaciones en Humanidades y

Ciencias Sociales, IdIHCS (UNLP-CONICET)

Facultad de Humanidades y Ciencias de la Educación

Universidad Nacional de La Plata

Argentina

geraldine.rogers@gmail.com

ORCID: 0000-0002-3443-1779

Fecha de recepción: 30/06/2021 | Fecha de aceptación: 27/07/2021

Resumen: Las publicaciones periódicas emergieron como un campo de interés crítico a fines del siglo pasado. Este interés adquirió distintos matices en los años siguientes: se acentuó el análisis en su importancia para la historia intelectual, los vínculos entre literatura, política y mercado, los circuitos de consumo de las publicaciones en tanto bienes culturales y su materialidad como objetos impresos, mediáticos y visuales. En este artículo se comparan estas tendencias generales con los aportes publicados en las últimas décadas en la revista Anclajes y sus proyecciones.

Palabras clave: publicaciones; circulación; Argentina; siglo XX; siglo XXI. 
Abstract: Periodicals emerged as a field of critical interest at the end of the previous century. This interest was nuanced and covered many fields: for instance, the analysis of the links between literature, politics and the markets, as well as the circulation networks of publications as cultural goods and their materiality as printed, media and visual objects. This article compares these general trends with the contributions published in recent decades in Anclajes and its projections.

Keywords: publications; circulation; Argentina; XX century; XXI century.

\section{Anclajes: contribuiçóes para a agenda crítica de estudos em periódicos}

Resumo: Os periódicos surgiram como campo de interesse crítico no final do século passado. Este interesse adquiriu nuances diferentes nos anos seguintes: acentua-se a análise das ligações entre a literatura, a política e o mercado, os circuitos de consumo das publicações como bens culturais e a sua materialidade como impressos, media e objectos visuais. Este artigo compara essas tendências gerais com as contribuições publicadas nas últimas décadas na revista Anclajes e suas projeções.

Palavras chave: publicações; circulação; Argentina; Século XX; Século XXI.

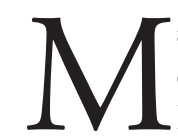
ás de veinte artículos y reseńas publicados a lo largo de un cuarto de siglo dan cuenta de los aportes de la revista del Instituto de Investigaciones Literarias y Discursivas de la Universidad Nacional de La Pampa a la agenda crítica de los estudios sobre publicaciones periódicas y áreas vinculadas: historia de la literatura, historia del libro y la edición, estudio de los géneros discursivos, historia intelectual, entre otras. Esas contribuciones muestran no solo la relevancia que fue adquiriendo el estudio de las publicaciones periódicas en los últimos años sino también los rasgos y tendencias de las investigaciones que las toman por objeto.

Varios artículos publicados por Anclajes entre 2003 y 2004 abordaron diarios y revistas argentinos como espacios discursivos, a partir del impulso y la renovación que por esos años fue adquiriendo la historia intelectual, con su particular interés por los impresos que ponen en circulación la palabra pública. Un artículo de Ana Rodríguez publicado en 2003 analizó la discursividad católica en la revista Criterio y los vínculos entre el discurso estatal y el discurso de la Iglesia. Con herramientas del análisis crítico del discurso (inspirado en las formulaciones de Mijail Bajtin y Michel Pêcheux) estudió la ideología de esa publicación como tribuna de sectores conservadores argentinos. Atenta a la relación entre las contradicciones del campo social y "el campo semiótico", buscó indagar cómo "estos conflictos dejaron su huella en las páginas de la revista dando lugar a una interdiscursividad en la que operan el discurso católico, la retórica peronista y el discurso científico". 
En un artículo del año siguiente, Verónica Delgado investigó la revista $\mathrm{La}$ Nota (Buenos Aires, 1915-1920) "como espacio discursivo fundamental para focalizar y estudiar las reconfiguraciones del campo literario argentino de esos ańos". Planteó las relaciones y formas de articulación de la literatura con el campo político en un contexto nacional caracterizado por la relativa democratización abierta por la ley de sufragio "universal" y en un marco internacional signado por la Primera Guerra Mundial, lo que explica ciertos posicionamientos enunciados desde sus páginas. Buscando contribuir a pensar los vínculos entre literatura, política y mercado incorporó también, en relación con este último, algunos aspectos que irían adquiriendo creciente importancia en los estudios sobre publicaciones periódicas de los años siguientes: las ilustraciones, las formas de venta, los avisos publicitarios y otros aspectos de su materialidad como objetos impresos y como productos que formaron parte del mercado de bienes culturales.

También desde la historia literaria, y más específicamente desde el interés por la emergencia del género novelístico en los diarios argentinos de fines del siglo XIX, en 2005 Fabio Espósito abordó los folletines del diario Sud-América como novelas de la élite en la prensa política de 1880 . Se propuso analizar las operaciones de intervención facciosa del órgano de prensa dirigido por Paul Groussac, mediante estrategias desplegadas en varias secciones del diario. Entre ellas el folletín, donde el uso del género novelístico se transformó en una herramienta política más para fortalecer la línea editorial de la publicación, con objetivos político-económicos bien definidos. El caso estudiado - un folletín novelesco cuyo autor era nada menos que el director del diario- mostró el papel del periódico como dispositivo de enunciación literaria subordinado a las estrategias políticas y económicas de la élite.

Otra zona de la cultura impresa, también de fines del siglo XIX argentino, fue abordada más recientemente por María Vicens en un estudio de 2017 sobre literatura, mujer y trabajo en la prensa de Buenos Aires. En sintonía con la centralidad de las problemáticas de género, se ocupó de las intervenciones de dos escritoras, Josefina Pelliza y Lola Larrosa, en periódicos como La Alborada del Plata, de cuya dirección se hicieron cargo respectivamente en distintos momentos de la vida del semanario. Este artículo, como otros ya mencionados, muestra a la prensa como el instrumento y espacio fundamental donde es posible leer configuraciones emergentes. En efecto, en estas publicaciones periódicas se hicieron presentes los primeros debates en torno a la autoría femenina y su posible vínculo con el mundo del trabajo. Ahí las mujeres con aspiraciones literarias dieron a conocer sus escritos y afirmaron sus trayectorias, convirtiéndolos en espacios relevantes en la negociación (siempre producto del conflicto y la tensión) de sus propias figuras autorales, calibrando las posibilidades efectivas de sus propias carreras como escritoras.

Centrado en un período anterior de la cultura argentina, la década de 1830, el artículo de María Laura Romano abordó los periódicos y los "sueltos" de Luis Pérez, en una línea de investigación que tiene una tradición propia, los estudios sobre 
la prensa del siglo XIX. Este trabajo, publicado en Anclajes hace dos años, muestra una de las tendencias emergentes del campo disciplinar, que consiste en atender a los rasgos materiales de las publicaciones, aquí en relación con el tipo de lector al que se buscaba interesar y con el sentido e ideología de los textos. Un artículo del año anterior, de Juan Ignacio Pisano, había abordado El Torito de los Muchachos, una gaceta de Pérez que intervino en la "guerra de palabras que buscaron ocupar un espacio público imaginado como territorio de conflictos discursivos".

En 2010 Griselda Fanese analizó el papel de una revista neuquina en la transición desde la última dictadura cívico-militar a la democracia. El estudio sobre Coirón explica cómo a través de esta publicación un grupo de escritores se posicionó en la esfera pública norpatagónica para modificar las condiciones de expresión poética y política, disputando un lugar en el diseño de políticas culturales.

De la serie de artículos que consideramos, un conjunto se dedica a analizar impresos que no son revistas, lo que constituye un dato significativo para el análisis. Como es sabido, desde el siglo XIX y a lo largo de todo el siglo siguiente, una parte fundamental de la literatura surgió en el marco de las publicaciones periódicas, cuyas condiciones de producción y recepción dejaron huellas significativas en las modalidades de la escritura literaria, la que a su vez fue aportando recursos para la transformación de aquellos, en un juego activo de interrelaciones. La importancia del conjunto de los impresos periódicos para la vida cultural fue creciente y contribuyó a la formación y difusión de propuestas y producciones literarias. Muchos escritores y escritoras, además de participar en las pequeñas revistas que fueron sus plataformas como artistas, se desempeñaron activamente en los diarios, donde tenía lugar la lectura a gran escala y donde accedieron a una visibilidad pública amplia y a grados variables de profesionalización. Sin embargo, aunque resulta evidente la tendencia creciente a releer y reconstruir la historia cultural latinoamericana a partir del estudio de las publicaciones periódicas, lo cierto es que en los estudios sobre el siglo XX el foco ha estado -y sigue estando todavía- en las revistas, y más específicamente en las llamadas "revistas culturales", quedando todo un continente de historia cultural y literaria aún por descubrir en las páginas de los diarios. Ese rasgo se intensifica sobre todo en los estudios sobre la literatura del siglo XX, cuya relación con los diarios no ha tenido todavía un desarrollo paralelo a la importancia del fenómeno. El artículo de María de los Ángeles Mascioto sobre Jorge Luis Borges como editor, publicado en 2018, resulta un aporte valioso en ese sentido, ya que muestra la articulación fundamental entre las diversas prácticas de un escritor que fue a la vez editor de libros, de revistas y del suplemento sabatino de un diario popular a partir de la década de 1930, poniendo en perspectiva relaciones múltiples donde las prácticas de edición y de escritura se solapan y realimentan mutuamente.

Antonia Viu y Claudia Darrigandi también tomaron como objeto de indagación el diario en un artículo de 2017 sobre los perfiles de artistas e intelectuales en textos de Georgina Durand, rescatando el trabajo de esta periodista chilena 
como entrevistadora del diario La Nación de su país. Las autoras reflexionan sobre los regímenes de lo decible, las tensiones entre lo público y lo privado, así como la potencial relación entre la periodista y sus lectores en las entrevistas, asignándoles un valor activo en el campo cultural a través de la construcción de ciertos perfiles biográficos de figuras de las artes y la cultura. En ese sentido, la prensa es considerada como espacio y archivo donde se articula cierta información relativa a la constitución de esos campos, las especificidades de cada saber, los cánones y las jerarquías particulares.

En un artículo de 2019 sobre las crónicas de viaje de Elías Castelnuovo, Esteban Da Ré encuentra un campo para indagar y complejizar el estudio de las relaciones entre los escritores y la política en la prensa. El análisis puso foco en las crónicas sobre la visita a la URSS en 1931, y en la polémica que ellas suscitaron en la revista Actualidad (uno de los espacios primeros de circulación de estos escritos antes de su posterior compilación en el volumen Yo vi...! ...en Rusia). El trabajo se inscribe en una línea que lee las características de una escritura y de una poética atendiendo tanto a sus circuitos de producción, circulación y recepción como a los vínculos entre literatura y política, con particular énfasis en las tensiones de la izquierda de la época.

Para quienes nos dedicamos al estudio de la literatura argentina del siglo XX, Cursos y Conferencias es el nombre de la revista donde se publicó un ensayo famoso, producto de una conferencia, "El escritor argentino y la tradición" de J. L. Borges. En un número reciente de Anclajes Ornela Lizalde desplegó varios aspectos de esa revista editada por el Colegio Libre. La investigación se centró en la etapa específica (1941-1952) en que, bajo la dirección de Arturo Frondizi, la publicación se orientó a diversas formas de intervención pública en un período caracterizado por una compleja relación entre los intelectuales, la sociedad y el Estado.

Un artículo de María Terán sobre la escritura de crónicas y testimonios a propósito de la matanza de Tlatelolco pone en evidencia interacciones productivas entre literatura y prensa. Interesa sobre todo el análisis de los procedimientos verbales y la inclusión de materiales visuales empleados por Elena Poniatowska en sus testimonios y por Carlos Monsiváis en sus crónicas, así como las conexiones y trasformaciones a partir de las noticias periodísticas abocadas a los mismos acontecimientos.

Desde hace algunos años, Anclajes destina un espacio a las entrevistas. Una de ellas, realizada en 2018 por María Carolina Domínguez a Andrea Pagni lleva por subtítulo "revistas culturales, género y plurilingüismo". El diálogo señala una perspectiva de investigación en los estudios literarios centrada en la traducción, como problema o proceso que, en ciertos momentos y procesos históricos latinoamericanos, tiene entre sus agentes más destacados a las revistas culturales y literarias en su rol de mediadoras e importadoras. El interés en la traducción conduce a examinar los cruces entre historia literaria, publicaciones periódicas y edición de libros. 
Las reseñas también fueron una zona de actualización sobre el campo de estudios en expansión. En su segundo número de 1998 Anclajes publicó una nota bibliográfica de Marisa Elizalde sobre la edición a cargo de la Universidad Nacional de Quilmes del periódico que, según advertía en su encabezamiento hace casi exactamente un siglo "aparece cuando puede y por suscripción voluntaria": La voz de la mujer. Periódico comunista - anárquico 1896 -1897. En 2008 Rosario Pascual Battista reseñó el libro Geopolíticas de la cultura finisecular en Buenos Aires, París y México: las revistas literarias y el modernismo editado por el Instituto Internacional de Literatura Iberoamericana de Pittsburgh. Al año siguiente, Daniela Rut Melchor presentó La epopeya de una generación y una revista. Las redes editoriales de José Carlos Mariátegui en América Latina de Fernanda Beigel. En 2014 María Pía Bruno reseñó Cuando lo nuevo conquistó América. Prensa, moda $y$ literatura en el siglo XIX de Víctor Godgel. El siguiente año aparecieron dos reseñas de actualización bibliográfica en torno a estos temas: una sobre Almacenes de un tiempo en fuga. Revistas culturales en la modernidad hispánica, a cargo de María de los Ángeles Mascioto, y otra de Ana Eugenia Vázquez sobre Sarmiento, redactor y publicista. Con textos recobrados de El Progreso (1842-1845) y La Crónica (1849-1850) de Hernán Pas. En 2018 Cristina B. Fernández presentó su lectura de Cuando la ciencia despertaba fantasias. Prensa, literatura y ocultismo en la Argentina de entresiglos de Soledad Quereilhac y, en 2019, Julieta Viu Adagio reseńó Crónicas travestis sobre el periodismo de Alfonsina Storni, Clarice Lispector y María Moreno de Mariela Méndez.

Este recorrido es una muestra parcial pero contundente del aporte realizado por Anclajes a un área del conocimiento que se ha ido consolidando como un ámbito de renovación disciplinar para los estudios literarios.

En efecto, resulta indispensable revisar la perspectiva metodológica para el estudio de la literatura a partir de la relevancia que adquiere el contexto de circulación de los textos escritos, una línea en que hemos venido trabajando desde hace más de una década ${ }^{1}$. Desde esta perspectiva, los libros pierden su hegemonía como soporte casi único de la literatura, y los textos se muestran no sólo como formas estéticas sino también como objetos en cuya producción intervienen diversos agentes, además de los escritores: impresores, dibujantes, periodistas, editores, entre otros. Eso supone tomar en cuenta los aspectos mediáticos, materiales y visuales a través de los cuales los textos se ofrecieron a la lectura. Y desplaza el foco de los aspectos individuales a los rasgos supraindividuales, que dan cuenta de vínculos activos entre distintas prácticas y esferas de la vida social. Estudiar la literatura en las revistas, los diarios y los semanarios elaborados colectivamente obliga a pensar las interacciones y los grupos. En el terreno de

1 Desde 2013 venimos trabajando de manera colectiva en diversas iniciativas (coloquios, proyectos de investigación, ediciones) y desde 2020 conformamos el Programa Publicaciones Periódicas y Literatura (PPLit) radicado en el IdIHCS e integrado por proyectos de investigadores de la región. La perspectiva arriba citada pertenece a la fundamentación del programa y de las líneas de investigación que incluye: http://idihcs.fahce.unlp.edu.ar/pplit/ 
los estudios literarios definir las publicaciones como "contextos formativos de la literatura" significa, en primer término, subrayar e insistir en el carácter determinante de estas producciones colectivas dentro de un proceso cultural y, en particular, respecto de una práctica especializada. Al mismo tiempo, esta noción de contexto importa la necesidad de explorar las relaciones entre las publicaciones y la escritura, precisando cómo las publicaciones periódicas a la vez determinaron, produjeron y fueron espacio de configuración de ciertos tipos y géneros de escritura o poéticas particulares, de lectores y prácticas de lectura, de figuras de escritores y de formas de profesionalidad y de sociabilidad ligadas con ellas, así como también de tradiciones críticas, de formas de publicidad, de circulación y de exhibición de los textos. Esta perspectiva produce necesariamente algunas transformaciones y desplazamientos en la investigación sobre literatura, especialmente la necesidad de revisar las modalidades, preguntas y problemas para la historia literaria, y principalmente el imperativo de pensar en las interacciones y la mutua afectación entre prácticas, normalmente no considerada. Las miradas provenientes de otras áreas resultan indispensables para el estudio de objetos y problemas que requieren del diálogo interdisciplinar.

Anclajes, la revista del IILyD, ha sido y es una importante difusora de avances de investigación sobre este campo en expansión donde convergen intereses multidisciplinares que desde hace un tiempo están abriendo líneas de investigación de relevancia indudable.

\section{Referencias bibliográficas}

Bruno, María Pía. "Reseña de Cuando lo nuevo conquistó américa. Prensa, moda y literatura en el siglo XIX de Víctor Goldgel". Anclajes, vol. 18, nº. 1, 2014, pp. 87-89.

Da Ré, Esteban Virgilio. "Las crónicas de viaje a la URSS de Elías Castelnuovo: el humor como estrategia crítica". Anclajes, vol. XXIII, n. ${ }^{\circ}$ 2, mayo-agosto 2019, pp. 21-38, https://doi.org/10.19137/anclajes-2019-2322.

Darrigrandi, Claudia y Antonia Viu. "Perfiles de artistas e intelectuales en Mis entrevistas (1943) de Georgina Durand". Anclajes, vol. XXI, N³, setiembrediciembre 2017, pp. 43-60, https://doi.org/10.19137/anclajes-2017-2134.

Delgado, Verónica y Geraldine Rogers, editoras. Revistas, archivo y exposición. Publicaciones periódicas argentinas del siglo XX. La Plata, Facultad de Humanidades y Ciencias de la Educación - Universidad Nacional de La Plata, 2019.

Delgado, Verónica, Mailhe, Alejandra y Geraldine Rogers, editoras. Tramas impresas. Publicaciones periódicas argentinas $(X I X-X X)$. La Plata, Serie Estudios e Investigaciones, Facultad de Humanidades y Ciencias de la Educación de la Universidad Nacional de La Plata, 2014. 
Delgado, Verónica y Geraldine Rogers. "Introducción”. Tiempos de papel. Publicaciones periódicas argentinas $(X I X-X X)$. La Plata, Facultad de Humanidades y Ciencias de la Educación de la Universidad Nacional de La Plata, 2016, pp. 8-12.

Delgado, Verónica. "Reconfiguraciones de debates y posiciones del campo literario argentino en el Semanario La Nota 1915-1920". Anclajes, vol. Vll. no 8, diciembre 2004, pp. 81-99.

Domínguez, María Carolina. "La traducción literaria, 'un laboratorio de escritura’: revistas culturales, género y plurilingüismo. Entrevista a Andrea Pagni”. Anclajes, vol XXII, no 3, setiembre-diciembre 2018, pp. 119-135, https:// doi.org/10.19137/anclajes-2018-2239.

Elizalde, Marisa. "Reseña de La voz de la mujer. Periódico comunista - anárquico 1896 -1897”. Anclajes, vol. II, n. ${ }^{\circ} 2$, 1998, pp. 109-112.

Espósito, Fabio. "Los folletines del diario Sud-América. Las novelas de los patricios en la prensa política de 1880". Anclajes, vol. IX, n. ${ }^{\circ}$ 9, 2005, pp. $39-52$.

Fanese, Griselda. "La revista Coirón (Neuquén, 1983): escribir en la política y la poética”. Anclajes, vol. 14, n. ${ }^{\circ}$ 14, 2010, pp. 73-89.

Fernández, Cristina Beatriz. "Reseña de Cuando la ciencia despertaba fantasías. Prensa, literatura y ocultismo en la Argentina de entresiglos de Soledad Quereilhac". Anclajes, vol. XXII, n. ${ }^{\circ}$ 1, enero-abril 2018, pp. 98-100, https://doi. org/10.19137/anclajes-2018-2216.

Lizalde, Ornela. "Cursos y Conferencias: la revista del Colegio Libre bajo la dirección de Arturo Frondizi (1941-1952)". Anclajes, vol. XXV, n. ${ }^{\circ}$ 2, mayoagosto 2021, pp. 213-232, https://doi.org/10.19137/anclajes-2021-25214.

Mascioto, María de los Ángeles. "Borges editor". Anclajes, vol. XXII, n. ${ }^{\circ}$ 2, mayo-agosto 2018, pp. 57-68, http://doi.org/10.19137/anclajes-2018-2224.

Mascioto, María de los Ángeles. "Reseña de Almacenes de un tiempo en fuga. Revistas culturales en la modernidad hispánica de Hanno Ehrlicher/ Nanette Rißler- Pipka (eds.)”. Anclajes, vol. XIX, n. ${ }^{\circ}$ 1, 2015, pp. 81-83.

Melchor, Daniela Rut. Reseña de La epopeya de una generación y una revista. Las redes editoriales de José Carlos Mariátegui en América Latina de Fernanda Beigel. Anclajes, vol. 13, n 13, 2009, pp. 187-189.

Pascual Battista, Rosario. "Reseña de Geopoliticas de la cultura finisecular en Buenos Aires, París y México, las revistas literarias y el modernismo de Adela Pineda Franco". Anclajes, vol. 11, n. ${ }^{\circ}$ 12, 2008, pp. 234-235.

Pisano, Juan Ignacio. "El Torito de los Muchachos: lectura de confesiones, escenario de opiniones". Anclajes, vol. XX, n 2, mayo-agosto 2016, pp. 51-63, https://doi.org/10.19137/anclajes-2016-2024. 
Rodríguez, Ana. "Cuerpo, familia y género. La Revista Criterio, discurso católico en la Argentina de mediados del siglo XX". Anclajes VII, n. ${ }^{\circ}$ 7, diciembre 2003, pp. 201-240.

Rogers, Geraldine. "Publicaciones periódicas del siglo XX: aspectos emergentes, miradas latinoamericanas". Catedral Tomada. Revista de Crítica Literaria Latinoamericana vol. 6, n. ${ }^{\circ}$ 11, 2018, pp. 1-12, https://doi.org/10.5195/ ct/2018.375.

Romano, María Laura. "Espacios escritos. El formato de los periódicos y los sueltos de Luis Pérez (1833). Anclajes, vol. XXI, n 2, mayo-agosto 2017, pp. 41-57, https://doi.org/10.19137/anclajes-2017-2123.

Vázquez, Ana Eugenia. "Reseña de Sarmiento, redactor y publicista. Con textos recobrados de El Progreso (1842-1845) y La Crónica (1849-1850) de Hernán Pas". Anclajes, vol. 19, n. ${ }^{\circ}$ 2, 2015, pp. 74-76.

Vicens, María. "Ensayos profesionales: literatura, mujer y trabajo en la prensa porteńa finisecular". Anclajes, vol. XXI, n², mayo-agosto 2017, pp. 77-94, https://doi.org/10.19137/anclajes-2017-2125.

Viú Adagio, Julieta. "Reseña de Crónicas travestis. El periodismo transgresor de Alfonsina Storni, Clarice Lispector y María Moreno de Mariela Méndez". Anclajes, vol. XXIII, n. ${ }^{\circ}$ 2, mayo-agosto 2019, pp. 103-104, https://doi. org/10.19137/anclajes-2019-2328. 